\title{
Significant Otorhinolaryngologic Case Reports, and Its Impacts
}

\section{Samia Ahmed Fawaz*}

Ain Shams University, Cairo, Egypt

\section{Case of Nonalcoholic Steatohepatitis Occurring in Combination with OSA}

Who Showed Improvement of Liver Function by Continuous Positive Airway Pressure; this issue is very agonizing as with the increasing incidence of obesity and related disorders, it is thought that the number of patients with NASH will increase further and more patients with these conditions will be encountered in daily medical practice, that gives an alarming to pick up such cases and also considering that the association between OSAS and NASH remains poorly understood, which need further evaluation of such cases.

\section{New Sinusitis Associated Syndrome with Psoriasis and Periophthalmitis}

Which is an indicator of Sinusitis as a trigger of autoimmune inflammation, and also to consider the other factors predisposing unexplained sinusitis, such as Wegener's granulomatosis, Churg-Strauss syndrome and, rarely, Guillain-Barre syndrome and fibromyalgia, which rising a question; is it a predisposing factor of sinusitis or as a result of active sinusitis with staphylococcus microbial flora, which was proposed to be a trigger of autoimmune inflammation, or consider it as an unknown autoimmune syndrome involving psoriasis, periophthalmitis and pansinusitis (PPP syndrome?, which need further collection of cases and explanation.

\section{A Rare Laryngeal Trauma: Complete Cricolaryngeal Separation}

As Laryngeal trauma is rare but life threatening injury, so High index of suspicion is required for diagnosis of such fatal injury if not diagnosed and treated. And to high light on the first priority, which is to establish and maintain stable airway, followed by examination and investigation of laryngeal injury and then plan of management. Also this issue spotlights on the importance of proper timing and adequate surgical repair which are vital for treating such life threatening lesions. 